\title{
Meteorites - The Significance of Collection and Curation and Future Developments
}

\author{
Caroline Smith
}

The Natural History Museum

email: c.l.smith@nhm.ac.uk

\begin{abstract}
Meteorites are some of the most important and valuable rocks available for scientific study. Approximately 43,000 meteorites are known on Earth and are egeologicalf samples of extraterrestrial bodies - meteorites are known to originate from asteroids, the Moon, Mars and possibly comets. With expanding exploration of our Solar System, meteorites provide the eground truthf to compare data collected by robotic missions with results gained from a variety of more accurate and precise techniques using laboratories on Earth. This talk will give an introduction to the history of meteorite science and the importance of meteorite collections to the field of meteoritics, planetary and solar system science. Curation of extraterrestrial samples is a particularly pertinent issue, especially with regards to particularly rare samples such as those from Mars like the recent Tissint meteorite. Future sample return missions to asteroids and Mars also pose siginificant challenges around the curation of these precious materials. Issues surrounding the curation of samples and how curation and curatorial actions can influence scientific studies will also be discussed.
\end{abstract}

